\title{
Through thick and thin: dual regulation of insect flight muscle and cardiac muscle compared
}

\author{
Belinda Bullard ${ }^{1} \cdot$ Annalisa Pastore ${ }^{2}$
}

Received: 22 May 2019 / Accepted: 2 July 2019 / Published online: 10 July 2019

(c) The Author(s) 2019

\begin{abstract}
Both insect flight muscle and cardiac muscle contract rhythmically, but the way in which repetitive contractions are controlled is different in the two types of muscle. We have compared the flight muscle of the water bug, Lethocerus, with cardiac muscle. Both have relatively high resting elasticity and are activated by an increase in sarcomere length or a quick stretch. The larger response of flight muscle is attributed to the highly ordered lattice of thick and thin filaments and to an isoform of troponin $\mathrm{C}$ that has no exchangeable $\mathrm{Ca}^{2+}$-binding site. The $\mathrm{Ca}^{2+}$ sensitivity of cardiac muscle and flight muscle can be manipulated so that cardiac muscle responds to $\mathrm{Ca}^{2+}$ like flight muscle, and flight muscle responds like cardiac muscle, showing the malleability of regulation. The interactions of the subunits in flight muscle troponin are described; a model of the complex, using the structure of cardiac troponin as a template, shows an overall similarity of cardiac and flight muscle troponin complexes. The dual regulation by thick and thin filaments in skeletal and cardiac muscle is thought to operate in flight muscle. The structure of inhibited myosin heads folded back on the thick filament in relaxed Lethocerus fibres has not been seen in other species and may be an adaptation to the rapid contractions of flight muscle. A scheme for regulation by thick and thin filaments during oscillatory contraction is described. Cardiac and flight muscle have much in common, but the differing mechanical requirements mean that regulation by both thick and thin filaments is adapted to the particular muscle.
\end{abstract}

Keywords Insect flight muscle $\cdot$ Cardiac muscle $\cdot$ Troponin $\cdot$ Thick filament regulation

\section{Introduction}

Robert Hooke (1665) observed the high frequency oscillations of the haltere in Diptera and recognised their importance in keeping insects with high wing beat frequency in balance during flight (Fig. 1). He also appreciated that wing beat frequency could be estimated by the sound. The haltere is a modified hindwing in the Diptera. It was nearly 300 years before Pringle (1949) showed that the haltere muscle of another fly (Calliphora) is stimulated to contract at $100-150 \mathrm{~Hz}$ by resonant oscillations of the thorax, independently of any repetitive input from thoracic nerves. The result with the haltere led Pringle to investigate the flight muscles of Calliphora, and he found that the oscillating

Belinda Bullard

belinda.bullard@york.ac.uk

1 Department of Biology, University of York, York YO10 5DD, UK

2 The Wohl Institute, King's College London, 5 Cutcombe Road, London SE5 9RT, UK contractions are also activated by changes in the shape of the thorax (Pringle 1949). The opposing dorsal longitudinal and dorso-ventral muscles are attached directly to the thoracic cuticle; when one muscle contracts, the other experiences a rapid stretch, due to distortions in the shape of the thorax. After a short delay, the stretched muscle contracts during the release phase of the cycle. Stretch-activation (SA) of flight muscle enables the wingbeat frequencies of flies to reach $200 \mathrm{~Hz}$, without rapid changes in $\mathrm{Ca}^{2+}$ levels. The majority of flying insects (75\%) have indirect flight muscle (IFM) activated by stretching (Josephson et al. 2000). The flight muscles of the giant waterbug, Lethocerus, have been used to study the structure and function of IFM. Another early microscopist and contemporary of Hooke's, the Dutch van Leeuwenhoek (1719), was the first to see branched fibres and banded patterns in heart muscle. 
Fig. 1 Observations made by Robert Hooke on a fly. This paragraph is taken from a summary of Hooke's observations published in Micrographia: Or, Some Physiological Descriptions of Minute Bodies Made by Magnifying Glasses. With Observations and Inquiries Thereupon (Hooke 1665)

\section{A Fly's Wing.}

Our Author tells us, (having made many Trials to find out after what manner the vibrative Motions of a Fly's Wings are performed) that the extreme Limits of the Vibrations were ufually about the Length of the Body diftant from one another, tho' often Morter, and fometimes longer: That commonly the foremoft Limit was a little above the Back, and the hinder fomewhat below the Belly; between which two Limits, if one may guefs by the Sound, the Wing feemed to move backwards and forwards with an equal Velocity: And thefe Vibrations between the two Limits are fo fwift, that 'tis very likely it makes many hundreds, if not thoufands, of Vibrations in a Second of Time; fo that probably the Wing of a Fly is one of the quickeft Vibrations in the World.

$M$ is a little Body, like in Appearance to a long hanging Drop of fome tranfparent vifcid Fluid. This is one of the Ballances or Poifes which moft Kinds of Flies that have only two Wings are furnilhed with. It grows out juft under the hinder Part of the Root of the Wing, and may be obferved conftantly to move before it. The Ufe of thefe Poifes is undoubtedly to keep the Body fteady and upright in flying; for if one of them be cut off, the Infeet will fly as if one Side was over-balanced, and ere long tumble to the Ground, and if both be taken away, its Flight is aukward and unfteady, maniferting the Want of fome neceffary and effential Part.

\section{Dual regulation}

IFM and cardiac muscle have much in common. Both perform rhythmic contractions and both are noticeably activated by an increase in sarcomere length (SL), or by a quick stretch. However, the way in which similar outcomes are achieved often differs in the two types of muscle. Each heart beat is activated by a pulse of $\mathrm{Ca}^{2+}$ ions, and the muscle relaxes when $\mathrm{Ca}^{2+}$ is removed. Although $\mathrm{Ca}^{2+}$ is necessary for the oscillatory contractions of IFM, the concentration remains nearly constant as the force fluctuates; the stimulus for contraction is stretch, not $\mathrm{Ca}^{2+}$ (Pringle 1978).

The geometry of the filament lattice in IFM is an important factor in the stretch-activation mechanism. Thin filaments are placed midway between two thick filaments, whereas in cardiac and skeletal muscle, each thin filament is equidistant from three thick filaments. Actin in the thin filaments of IFM has a half pitch of $38.7 \mathrm{~nm}$, compared to $36.0 \mathrm{~nm}$ in vertebrate actin (Reedy and Reedy 1985; ALKhayat et al. 2003). The longer half pitch in IFM is not an intrinsic property of the actin, but is likely to be due to the constraining influence of other thin filament proteins (Ruiz et al. 1998). The periodicity of troponin (Tn) on the thin filament is also $38.7 \mathrm{~nm}$ and all Tns on the filament have the same azimuthal orientation relative to the two neighbouring thick filaments. This is not the case for skeletal and cardiac muscle, where the $38.7 \mathrm{~nm}$ periodicity of the Tn does not match the half pitch of actin in the thin filament. In IFM, there are target zones every $38.7 \mathrm{~nm}$ on the thin filament that are favourably placed for interaction with myosin heads projecting from the thick filament (Tregear et al. 2004; Wu et al. 2010). The target zones are two actin monomers on each side of the long pitch actin helix; they are midway between the Tns so that targets and Tns alternate on the thin filament (Schmitz et al. 1994; Reedy et al. 1994; Wu et al. 2010; Bullard and Pastore 2011).

Contraction of striated muscle is under dual control by the thin filament and the thick filament. According to the steric blocking model, in relaxed skeletal and cardiac muscles, tropomyosin $(\mathrm{Tm})$ on the thin filament is held in a blocking position on actin by Tn, which prevents binding of myosin crossbridges. Troponin has three subunits: TnT, binds the complex to tropomyosin, $\mathrm{TnI}$ binds to $\mathrm{TnT}$ and to the $\mathrm{Ca}^{2+}$ binding subunit, $\mathrm{TnC}$; $\mathrm{TnI}$ also binds to actin in relaxing conditions. When $\mathrm{Ca}^{2+}$ binds to $\mathrm{TnC}$ in the active muscle, $\mathrm{TnI}$ is released from actin, and tropomyosin shifts so that myosin binding sites are partially exposed. Myosin binds co-operatively to the thin filament, the binding sites become fully open and the muscle contracts (McKillop and Geeves 1993; Gordon et al. 2000). Recent work has shown that in relaxed muscle, myosin heads bind to each other and to the thick filament backbone in an inhibited head motif (IHM) that maintains the filaments in an OFF state (Woodhead et al. 2005; Alamo et al. 2008; Zoghbi et al. 2008; Sulbaran et al. 2013; Hu et al. 2016). The OFF state is characterised by low ATPase activity and is also known as the super relaxed (SRX) state (Stewart et al. 2010). The majority of myosin heads are inhibited from binding to actin until thick filaments experience a force and heads in the IHM are released to the ON state; the number of heads turned ON is proportional to the force (Reconditi et al. 2014; Linari et al. 2015; Fusi et al. 2016; Irving 2017; Reconditi et al. 2017). 


\section{Length-dependent activation and stretch-activation}

IFM has greater passive tension than cardiac muscle and both are stiffer than skeletal muscle (Kulke et al. 2001; Neagoe et al. 2003; Miller et al. 2004; Linke 2007; Fukuda et al. 2008). Passive tension is due to proteins containing tandem Ig-domains that span the I-band, linking the ends of thick filaments to the Z-disc (Labeit et al. 1992; Trinick 1996); Houmeida et al. 2008). IFM fibres shorten by only about $3 \%$ during oscillatory contractions and the sarcomeres have exceptionally short I-bands. Kettin, which is largely responsible for passive tension in IFM, has tandem Ig-domains but no extensible sequence; it is attached to the ends of thick filaments, but extends no further along the filaments (Kolmerer et al. 2000; Kulke et al. 2001; Leake et al. 2003; Bullard et al. 2005). Titin in cardiac and skeletal muscles spans the half sarcomere from the $\mathrm{Z}$-disc to the M-line and in the A-band it is bound to the thick filament. The I-band region of cardiac titin has fewer Ig-domains and less extensible sequence than the skeletal isoforms (Cazorla et al. 2000; Neagoe et al. 2003). A relatively inextensible sarcomere means force is rapidly transmitted to the thick and thin filaments when the fibre is stretched. Kettin appears to have the sole function of maintaining the high stiffness of IFM, whereas titin has multiple functions. Cardiac titin can have varying stiffness, depending on the demands of the heart; phosphorylation and signalling through ligands bound to titin domains adapt the molecule to conditions (Krüger and Linke 2011; Linke 2018). Titin also determines the length of the thick filament (Tonino et al. 2017). Lethocerus IFM thick filaments have a core of paramyosin, which would reduce the compliance of the filaments and contribute to the greater stiffness of the sarcomere compared to cardiac muscle.

Length-dependent activation (LDA) underlies the Frank-Starling mechanism in which the force developed by the ventricular muscle of the heart during systole increases at longer sarcomere lengths; the sarcomere length depends on the extent of ventricular filling during diastole (de Tombe et al. 2010). A stiff titin is essential for LDA (AitMou et al. 2016). The increased force is due to a greater sensitivity of the contractile elements to $\mathrm{Ca}^{2+}$. In Fig. 2, isometric force developed by ventricular myocardium and IFM with increasing $\mathrm{Ca}^{2+}$ concentrations at different sarcomere lengths is compared. Cardiac muscle produces a relatively small increase in force when sarcomere length is increased by as much as $18 \%$; the $\mathrm{Ca}^{2+}$ sensitivity is only increased by $0.1 \mathrm{pCa}_{50}$ unit (Stelzer and Moss 2006) (Fig. 2a). For IFM, a much smaller increase in sarcomere length of $2 \%$ results in a $\mathrm{pCa}_{50}$ increase of about 1.0 unit (Fig. 2b). The cooperativity of the dependence of force on
$\mathrm{Ca}^{2+}$ concentration is greater in ventricular myocardium than in IFM, but in both cases, there is little change when the sarcomere length is increased. Both cardiac muscle and IFM have the potential to be activated above the level attained by fibres at rest length, either by an increase in sarcomere length, or by an increase in $\mathrm{Ca}^{2+}$ concentration. Clearly, the effect of sarcomere length is greater in IFM. LDA enables the ventricle to produce force during systole that varies depending on the filling of the ventricle during diastole. The effect of increased sarcomere length during diastole on systolic force is independent of the effect of load on the activation of thick filaments (Reconditi et al. 2017; Piazzesi et al. 2018).

Stretch activation (SA) and LDA both produce additional force, but probably by different mechanisms. A rapid stretch applied to a muscle that is already partially activated by $\mathrm{Ca}^{2+}$, results in a delayed rise in force; as the muscle shortens on release and the force decreases, relaxation is enhanced by a delayed shortening deactivation. At any particular muscle length, the force is greater during the shortening than during the lengthening phase, which produces a net output of work. SA is probably of minor importance in cardiac muscle compared to IFM. SA of isolated ventricular myocardium is greater at longer sarcomere lengths and it is suggested that when the myocardium is stretched during diastole, SA increases systolic force and delays relaxation. This would increase the volume of blood ejected at each heartbeat and is thought to increase the steepness of the Frank-Starling effect (Stelzer and Moss 2006; Campbell and Chandra 2006). The SA effect is relatively small: the maximum increase in delayed force after stretching isolated ventricular myocardium is about half the pre-stretch force (Stelzer et al. 2006a). This compares with a maximum SA of 3-5 times the pre-stretch force in Lethocerus IFM (Agianian et al. 2004; Linari et al. 2004).

\section{Modulating regulation}

Myosin-binding protein $\mathrm{C}$ (MyBP-C) affects the activation state of both thick and thin filaments in skeletal and cardiac muscle. The $\mathrm{C}$-terminal region of $\mathrm{MyBP}-\mathrm{C}$ is bound to the shaft of the thick filament; the N-terminal region extends from the thick filament and can bind either to the thin filament or to the $\mathrm{S} 2$ region of myosin. The interactions of the $\mathrm{N}$-terminal region depend on the level of phosphorylation (Pfuhl and Gautel, 2012; Craig et al. 2014). At low concentrations of $\mathrm{Ca}^{2+}$, N-terminal MyBP-C partially, or fully, activates the thin filament and inhibits the thick filament (Craig et al. 2014; Kampourakis et al. 2014; Mun et al. 2014; Harris et al. 2016). The effect of cardiac MyBP-C on myosin heads in the thick filaments of cardiomyocytes has been determined by measuring the proportion of heads in the SRX 

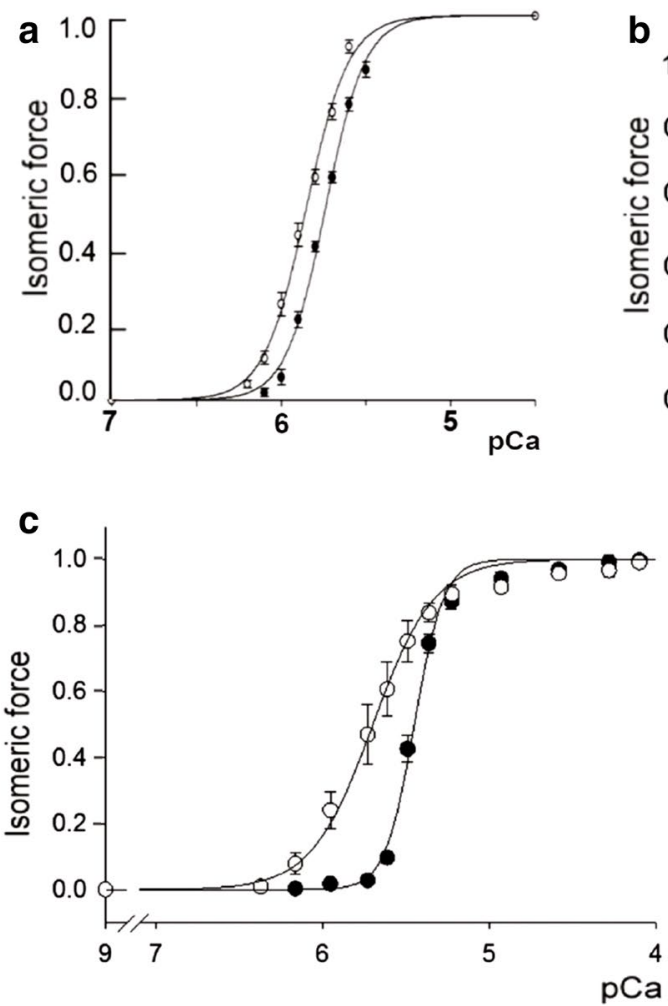

Fig. 2 Changes in the $\mathrm{Ca}^{2+}$ sensitivity of cardiac fibres and Lethocerus IFM. a and $\mathbf{b}$ The effect of SL. a Mouse ventricular myocardium at SL: filled black circles $1.9 \mu \mathrm{m}, \mathrm{pCa}_{50} 5.7, \mathrm{n}_{\mathrm{H}} 3.9$; open circles $2.25 \mu \mathrm{m}, \mathrm{pCa}_{50}$ 5.8, $\mathrm{n}_{\mathrm{H}}$ 3.6. From Fig. 1 in Stelzer and Moss (2006). b Lethocerus fibres at SL: filled blue circles $2.5 \mu \mathrm{m}, \mathrm{pCa}_{50} 6.2, \mathrm{n}_{\mathrm{H}} 2.1$; open red squares $2.53 \mu \mathrm{m}, \mathrm{pCa}_{50} 6.8, \mathrm{n}_{\mathrm{H}} 1.5$; open green diamonds $2.56 \mu \mathrm{m}, \mathrm{pCa}_{50}$ 7.2, $\mathrm{n}_{\mathrm{H}}$ 1.5. Mean $\pm \mathrm{SD}, \mathrm{n}=3-6$ from $1-3$ fibres. From R.J. Edwards and M. K. Reedy (unpublished). pCa scales in a and $\mathbf{b}$ are the same. $\mathbf{c}$ and $\mathbf{d}$ The effect of MyBP-C or TnC. $\mathbf{c}$ Rat ven-

state when MyBP-C is removed in homozygous knockout mice (McNamara et al. 2016). The decrease in the proportion of heads in the SRX state is consistent with increased disorder in the heads and disruption of the IHM in the knockout mice (Zoghbi et al. 2008). It is therefore likely that MyBP-C stabilises the IHM in the OFF state of the filament (Kampourakis et al. 2014; Irving 2017). As myosin heads bind to actin, MyBP-C may be displaced from the IHM and thick filaments turned ON. It has also been suggested that MyBP-C could be a stress sensor that disrupts the IHM in the OFF state of the thick filament (Harris 2016; Irving 2017; Piazzesi et al. 2018). The effect of a low concentration $\mathrm{N}$-terminal MyBP-C on the force developed by cardiomyocytes or trabeculae is to increase the $\mathrm{Ca}^{2+}$-sensitivity, so that the force $\mathrm{pCa}$ curve is shifted to lower $\mathrm{Ca}^{2+}$ concentrations; the cooperativity of force development is decreased (Fig. 2c) (Kampourakis et al. 2014; Harris et al. 2016). The function of MyBP-C may be to modulate the response of cardiac muscle to activation, effectively allowing a graded response to $\mathrm{Ca}^{2+}$ and an external force.

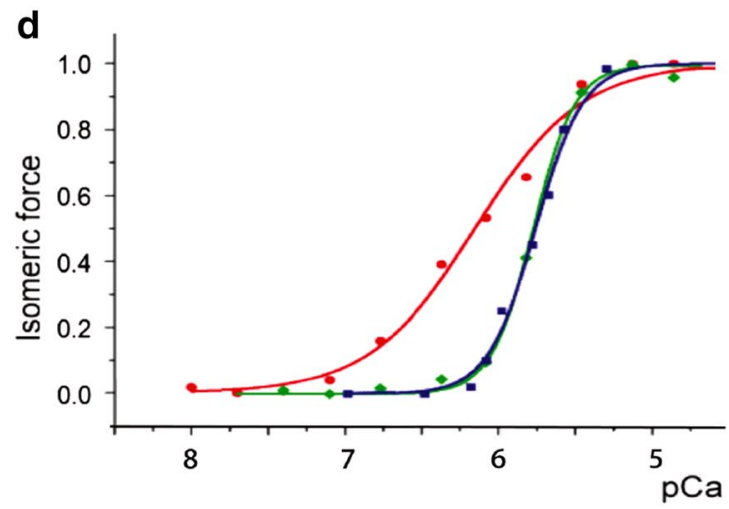

tricular trabeculae: filled black circles with no additions $\mathrm{pCa}_{50} 5.5, \mathrm{n}_{\mathrm{H}}$ 5.0 ; open circles with added MyBP-C fragment C1mC2 $(2 \mu \mathrm{M}) \mathrm{pCa}_{50}$ 5.7, $\mathrm{n}_{\mathrm{H}}$ 2.4. From Fig. 4 in Kampourakis et al. (2014). d Lethocerus IFM: filled red circles native fibres $\mathrm{pCa}_{50} 6.2, \mathrm{n}_{\mathrm{H}} 1.3$; filled green diamonds IFM in which $\mathrm{TnC}$ is replaced with $\mathrm{TnC}-\mathrm{F} 2 \mathrm{pCa}_{50} 5.8, \mathrm{n}_{\mathrm{H}} 3.2$; filled blue squares mouse ventricular myocardium SL $2.25 \mu \mathrm{m}, \mathrm{pCa}_{50}$ 5.8, $\mathrm{n}_{\mathrm{H}}$ 3.6. Lethocerus results are from Bullard and Leonard (unpublished), mouse result is from Fig. 1 in Stelzer and Moss (2006). pCa scales are the same in $\mathbf{c}$ and $\mathbf{d}$

The force developed by isolated Lethocerus IFM fibres has high $\mathrm{Ca}^{2+}$ sensitivity and low cooperativity, with a force-pCa curve similar to that of cardiac muscle with added N-terminal MyBP-C (Fig. 2d). IFM does not have MyBP-C and the characteristic dependence of force on $\mathrm{Ca}^{2+}$ concentration is due to the troponin complex in the flight muscle, which differs from the complex in skeletal and cardiac muscles (Bullard and Pastore 2011). The troponin has the subunits, TnT, TnH (TnI) and TnC. TnH has the sequence of TnI in the N-terminal half and a proline-alanine-rich sequence in the C-terminal half. There are two isoforms of $\mathrm{TnC}$ : the major isoform, $\mathrm{TnC}-\mathrm{F} 1$, has a single $\mathrm{Ca}^{2+}$-binding site in the $\mathrm{C}$-lobe and no exchangeable $\mathrm{Ca}^{2+}$ site in the $\mathrm{N}$-lobe; a minor isoform, TnC-F2 has one $\mathrm{Ca}^{2+}$-binding site in the $\mathrm{C}$-lobe and an exchangeable $\mathrm{Ca}^{2+}$ site in the $\mathrm{N}$-lobe. TnC-F1 is essential for stretch-activated contractions, whereas TnC-F2 promotes $\mathrm{Ca}^{2+}$-activated isometric contraction (Agianian et al. 2004; Krzic et al. 2010). The high $\mathrm{Ca}^{2+}$ sensitivity and low cooperativity of the IFM force-pCa curve is due to TnC-F1. When the $\mathrm{TnC}$ in fibres is replaced with TnC-F2 alone, the curve is 
the same as that of cardiac fibres that have been activated by stretching (Fig. 2d). Like TnC-F2, cardiac TnC (cTnC) has only one exchangeable $\mathrm{Ca}^{2+}$ site in the $\mathrm{N}$-lobe, (compared to the two exchangeable sites in skeletal TnC). Therefore, the force developed by IFM is restrained from cardiac-like $\mathrm{Ca}^{2+}$ dependence by a $\mathrm{TnC}$ that has no exchangeable $\mathrm{Ca}^{2+}$. The dominance of the effect of TnC-F1 over TnC-F2 in native fibres is likely to be due to the 9:1 stoichiometry. The high sensitivity of IFM to $\mathrm{Ca}^{2+}$ means that stretch-activated oscillatory contractions can occur at priming concentrations of $\mathrm{Ca}^{2+}$, where there is little isometric force. IFM and cardiac muscle bring about a similar change in $\mathrm{Ca}^{2+}$ sensitivity by different means.

Phosphorylation of myosin light chains modulates contraction in vertebrate skeletal and cardiac muscle and in insect flight muscle. The myosins have essential (ELC) and regulatory (RLC) light chains. Phosphorylation of the RLC in skeletal and cardiac muscles increases force at low concentration of $\mathrm{Ca}^{2+}$ and this is associated with an increase in disorder of myosin heads (Levine et al. 1996; Stelzer et al. 2006b; Stull et al. 2011; Kampourakis et al. 2016). The change in $\mathrm{Ca}^{2+}$ sensitivity is comparable to that obtained by an increase in sarcomere length, suggesting both RLC phosphorylation and LDA act in the same way to produce a more ON state of the thick filament.

The RLC of IFM myosin has an extended sequence at the $\mathrm{N}$-terminus, similar to that at the $\mathrm{N}$-terminus of cardiac myosin ELC. The cardiac ELC extension can bind to actin and is thought to maintain the optimal position of the myo$\sin$ head for production of force (Trayer et al. 1987; Lowey et al. 2007). The function of the RLC in IFM myosin has been investigated in living Drosophila and in isolated fibres (Tohtong et al. 1995; Dickinson et al. 1997; Moore et al. 2000; Farman et al. 2009). Mutant flies lacked the two phosphorylation sites in the RLC, or had the N-terminal extension truncated. In both cases, X-ray diffraction of the IFM showed decreased myosin mass associated with thin filaments, and myosin heads were more disordered in the phosphorylation mutants. The N-terminal extension of the RLC binds to actin and may align myosin heads with the target zones. Mechanics of the isolated IFM suggests that fewer crossbridges are recruited in phosphorylation mutants and electron micrographs of $\mathrm{Ca}^{2+}$-activated fibres are consistent with this. As in skeletal and cardiac muscle, phosphorylation of the RLC in IFM may increase the ON state of the thick filament, resulting in more available myosin heads. However, there was no significant effect on the $\mathrm{Ca}^{2+}$ sensitivity of isometric force developed by mutant IFM fibres (Dickinson et al. 1997). Mutant Drosophila could fly. The power output was reduced, but this was partially compensated for by an increase in wingbeat frequency and in the amplitude of the stroke. Therefore, RLC phosphorylation and an RLC extension are modulating factors, rather than being essential for flight.

\section{Lethocerus TnC and its interactions with $\mathrm{TnH}$ and Tnl}

TnC-F1 and F2 share $47 \%$ sequence identity and thus must have a similar fold. They could not be crystallized and were studied by solution nuclear magnetic resonance (NMR). $\mathrm{Ca}^{2+}$ binds in EF-hand 4 in TnC-F1 and in EF-hands 2 and 4 in TnC-F2 (Agianian et al. 2004). Their structure consists in a typical EF-hand fold with two globular domains spaced by a flexible linker. Each domain contains two EF-hand motifs, each of which comprises two $\alpha$-helices that flank an eleven residues loop, which is where $\mathrm{Ca}^{2+}$ binds when the loop contains six canonical residues. The two loops form the typical short antiparallel $\beta$-sheet that keeps the two EFhands side by side. A short $\mathrm{N}$ helix precedes the A helix, but it is formed only in some of the NMR structures of TnCF1 (De Nicola et al. 2007). As in all calmodulin-like folds, the two lobes are connected by a tethering helix which in some structures is a straight helix that confers a dumbbell shape to the protein (Herzberg and James 1985; Houdusse et al. 1997; Satyshur et al. 1988). In other X-ray and solution structures, the linker is disordered and unstructured, such as in TnC-F1 and TnC-F2 and cardiac TnC. This arrangement allows an undetermined relative orientation of the two lobes that can be instrumental in favouring interactions (Blumenschein et al. 2005; Dvoretsky et al. 2002; Slupsky and Sykes 1995; Sia et al. 1997; Vinogradova et al. 2005). The arrangement of TnC-F1 and -F2 is different and parallels the $\mathrm{Ca}^{2+}$-binding capacity of the two proteins. The $\mathrm{N}$ lobe of $\mathrm{TnC}-\mathrm{F} 1$ is in a closed conformation and has closest resemblance with the structure of the apo $\mathrm{N}$ lobe of skeletal TnC (1skt, Tsuda et al. 1999). Conversely, the holo C lobe of TnC-F1 shares highest similarity with the holo $\mathrm{C}$ lobe of human cardiac TnC in a complex with the inhibitory region of TnI (1ozs, Lindhout and Sykes, 2003). This implies that this lobe is in an open state and indicates that a single $\mathrm{Ca}^{2+}$ ion is sufficient to induce opening of the domain and that, already in its $\mathrm{Ca}^{2+}$-bound state, it is prepared to interact with $\mathrm{TnH}$. This is unlike EF hand 2 in the $\mathrm{N}$-lobe of cardiac $\mathrm{TnC}$, which also binds a single $\mathrm{Ca}^{2+}$, but is only in an open state when in complex with the switch region of TnI.

For comparison, both apo and holo forms of TnC-F2 are also structured in the absence of $\mathrm{Ca}^{2+}$ and have a comparable helical content. The role of $\mathrm{Ca}^{2+}$ is thus not structural. This is in contrast to the behaviour of the $\mathrm{C}$ lobe of skeletal and cardiac TnC. This lobe is unstructured in the absence of $\mathrm{Ca}^{2+}$ and acquires structure only in the presence of the cation (De Nicola et al. 2010). The dynamic properties of 
TnC-F1 and -F2 are overall similar (Sanfelice et al. 2016; De Nicola et al. 2007).

TnC-F1 and -F2 interact with TnH. TnC-F1 binds the $\mathrm{TnH}(30-61)$ peptide with a $\mathrm{K}_{\mathrm{d}}$ of $1.9 \mathrm{nM}$ and $0.9 \mathrm{nM}$ in the absence and presence of $\mathrm{Ca}^{2+}$ respectively. $\mathrm{TnH}(30-61)$ binds TnC-F2 with a $\mathrm{K}_{\mathrm{d}}$ of $8 \mathrm{nM}$ in the presence of $\mathrm{Ca}^{2+}$ and $40 \mathrm{nM}$ in the absence of $\mathrm{Ca}^{2+}$, or in the presence of the more weakly coordinated magnesium (Martin et al. 2011). The affinities of the TnH(126-159) peptide for TnC-F1 and TnC-F2 are comparable and around $13 \mu \mathrm{M}$ and $3 \mu \mathrm{M}$ in the absence and presence of $\mathrm{Ca}^{2+}$. Two-hybrid screening and NMR studies demonstrated that the N-terminus of $\mathrm{TnH}$ (residues 1-76) and the C-terminal lobe of TnC-F1 are necessary and sufficient for interaction of the two molecules (De Nicola et al. 2007). The N lobe of TnC-F1 does not bind to TnH. Consistent with these results, the NMR spectra of TnC-F1 in complex with $\mathrm{TnH}(30-61)$, or with the longer fragments $\mathrm{TnH}(1-224)$ and $\mathrm{TnH}(1-340))$ are similar, apart from the latter being broader because of the slower tumbling. This indicates that the region 30-61 of TnH is key for the interaction with TnC-F1. Likewise, TnC-F2 interacts with the peptides $\mathrm{TnH}(30-61)$ and $\mathrm{TnH}(126-159)$, designed by homology with regions involved in the interaction in vertebrates (De Nicola et al. 2007). The $\mathrm{C}$ lobe binds primarily $\mathrm{TnH}(30-61)$, whereas the $\mathrm{N}$ lobe interacts with TnH(126-159). This behaviour is similar to that observed in vertebrate troponin complexes.

\section{Experimentally based models of the Lethocerus Tn heterotrimer}

Modelling the structure of the Lethocerus Tn complex with TnC-F2 was attempted using the coordinates of the vertebrate cardiac complex (PDB ID: 1J1D) as template (De Nicola et al. 2007; Sanfelice et al. 2016). The model obtained recapitulates all the important features of the complex and provides an excellent starting structure for further studies (Fig. 3). The main problem encountered was found in TnT. Lethocerus $\mathrm{TnC}$ and $\mathrm{TnI}$ do not have major insertions or deletions compared to the vertebrate sequences. Standard homology modelling could thus be used to build their structures. In contrast, Lethocerus TnT has three large insertions, one in the middle of the first helix of the hairpin. The inserted residues were thus introduced by an iterative gap growing process alternated with a relaxation phase in a molecular dynamics calculation. In this way, the peptide chain could be lengthened progressively while maintaining the structure of the trimer in an energetically favourable state compatible with the template. The resulting TnT model suggests the presence of an insertion in the Lethocerus sequence which ends up with the hairpin loop. As a result, the overall shape of the complex remains similar but the orientation of

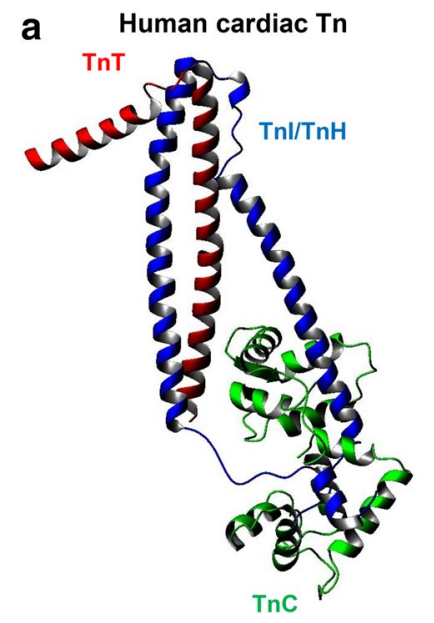

b Lethocerus $\mathrm{Tn}$

Fig. 3 Comparison of the X-ray structures of the human cardiac and Lethocerus IFM Tn complexes. a Human cardiac Tn (1J1D). b Lethocerus model of the Tn complex with TnC-F2, generated by homology, using cardiac Tn as a template. Lethocerus $\mathrm{TnC}$ and $\mathrm{TnH} / \mathrm{TnI}$ do not have major insertions or deletions; TnT however has three insertions in the Lethocerus Tn structure. The complex was modelled by an iterative gap-growing process in which residues were gradually added. After each insertion, the system was relaxed by molecular dynamics runs (Sanfelice et al. 2016). The degree of homology between TnC-F1 and TnC-F2 is so high that the Tn structures will be similar at this level of resolution

the N-terminal helix of TnT differs from that of the template, while keeping the pattern of intermolecular interactions with TnI and TnC unaltered (De Nicola et al. 2007). The similarity in the force-pCa curves for Lethocerus fibres substituted with TnC-F2 and cardiac fibres with cTnC (Fig. 2d), is consistent with the similarity in the structures of Lethocerus $\mathrm{Tn}$ with TnC-F2 and cardiac Tn (Fig. 3).

\section{Common features in regulation by TnC-F1 and cardiac TnC}

There are similarities between TnC-F1 and cTnC in how they affect the thin filament at a maximal concentration of $\mathrm{Ca}^{2+}$ (pCa 4.0-4.5). Equilibrium binding measurements with myosin S1 and regulated actin in Geeves's laboratory, using thin filaments with the Lethocerus Tm-Tn complex and $\mathrm{TnC}-\mathrm{F} 1$, showed that thin filaments regulated by $\mathrm{TnC}-$ $\mathrm{F} 1$ were about equally in the open and closed states at $\mathrm{pCa}$ 4.5 (Boussouf et al. 2007). This is in contrast to the effect of the troponin complex with TnC-F2, where filaments were $15 \%$ open and $78 \%$ closed at pCa 4.5 . Recently, the activation state of cardiac thin filaments has been estimated by stopped-flow kinetic measurements of Pi release from the thin filament-myosin-ADP-Pi complex. Cardiac thin filaments were activated by $\mathrm{Ca}^{2+}$ to $~ 70 \%$ of the maximum level, without binding myosin heads (Risi et al. 2017). The 


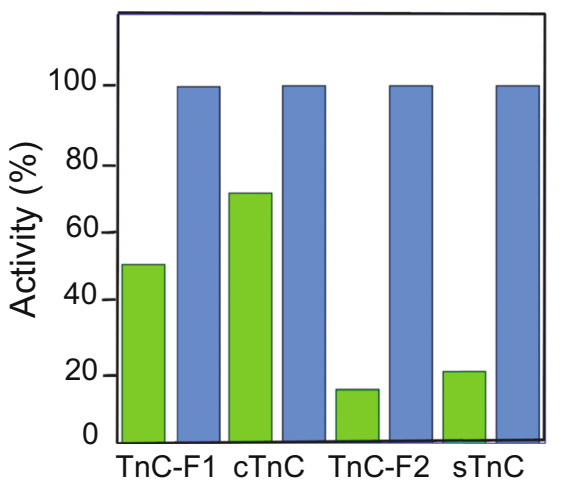

Fig. 4 Activation of cardiac and IFM thin filaments by TnC. The extent of activation of thin filaments by $\mathrm{Ca}^{2+}$ and by myosin $\mathrm{S} 1$ was estimated in solution (see text). Values are normalised to the maximum level of activation. IFM thin filaments have actin and Lethocerus $\mathrm{Tm}-\mathrm{Tn}$ with $\mathrm{TnC}-\mathrm{F} 1$ or TnC-F2. Cardiac thin filaments were isolated from pig hearts. Skeletal thin filaments were reconstituted from actin, Tm and Tn. Myosin S1 was from rabbit skeletal muscle. Green bars are the proportion of filaments in the open state at $\mathrm{pCa}$ 4.0-4.5; blue bars represent the maximum activity produced by $\mathrm{Ca}^{2+}$ and myosin S1. Thin filaments with Lethocerus TnC-F1 or TnC-F2 (Boussouf et al.2007); with cardiac TnC (cTnC) (Risi et al. 2017); with skeletal TnC (sTnC) (Heeley et al. 2006)

activation of IFM, cardiac and skeletal thin filaments by $\mathrm{TnC}$ are compared in Fig. 4. TnC-F1 and cTnC activate thin filaments to a $50-70 \%$ open state in the presence of $\mathrm{Ca}^{2+}$, although $\mathrm{TnC}-\mathrm{F} 1$ has no exchangeable $\mathrm{Ca}^{2+}$ in the N-lobe, while $\mathrm{cTnC}$ has one. Activation of IFM filaments by TnC$\mathrm{F} 2$ is similar to that of skeletal $\mathrm{TnC}$ (sTnC). In the case of IFM, activation at this high concentration of $\mathrm{Ca}^{2+}$ would not be physiological because in the native fibre, TnC-F2 would dominate the effect of TnC-F1. As discussed above, the structures of IFM and vertebrate Tn complexes are similar, although there are differences in the sequences of the subunits.

\section{Thick filament regulation}

Both cardiac and IFM thick filaments are regulated by the force applied to the filaments, although the structure and protein composition of the two are different. In cardiac and skeletal muscle thick filaments, three myosin heads emerge from the filament every $14.3 \mathrm{~nm}$. The spacing of these crowns changes from $14.3 \mathrm{~nm}$ in relaxed fibres to $14.5 \mathrm{~nm}$ on activation (Haselgrove 1975; Linari et al. 2015). In Lethocerus thick filaments, there are four myosin heads per crown (Reedy et al. 1981; Morris et al. 1991) and the spacing of crowns is $14.5 \mathrm{~nm}$ in both relaxed and activated fibres (Miller and Tregear 1972; Perz-Edwards et al. 2011).

The form of the IHM in relaxed fibres is similar in the striated muscle of several species and in cardiac muscle
(Woodhead et al. 2005; Zoghbi et al. 2008; Al-Khayat et al. 2013; Alamo et al. 2008; Sulbaran et al. 2013). Within a myosin molecule, a 'blocked' head binds to a 'free' head, which prevents the blocked head from binding to the thin filament, but leaves the actin-binding site of the free head available; the blocked head also contacts the $\mathrm{S} 2$ region of the same molecule and that of an adjacent molecule. The IHM in these muscles lies on the surface of the thick filament, forming a characteristic J shape. Activation of myosin heads on the thick filament is adjusted to the load on the filaments. X-ray diffraction measurements on cardiac trabeculae have shown that during diastole, the thick filament is in the OFF state, and during systole only some of the myosin heads are in the ON state; the proportion varies with the load on the filament, which is determined by the arterial pressure (Reconditi et al. 2017; Piazzesi et al. 2018). When cardiac muscle is activated, the change in the axial periodicity of the myosin heads from 14.3 to $14.5 \mathrm{~nm}$ is associated with a loss of helical order in the filament backbone. The conversion of myosin heads from the OFF state in the IHM to the ON state may be due to disruption of stabilising interactions with MyBP-C, as discussed above, However, there is not enough MyBP-C for a one-to-one interaction with myosin.

The IHM in Lethocerus IFM is unlike that in other muscles. Recently Taylor and colleagues (Hu et al. 2016) have determined the structure of the relaxed thick filament by cryo-electron microscopy. The shafts of myosin molecules in the filament backbone, as well as the position of additional proteins in the thick filament, have been resolved to 5.5-10 ̊. Candidates for the additional proteins are myofilin, which is not exposed to the surface of the filament, and flightin, which is on the surface (Ferguson et al. 1994; Qui et al. 2005). These additional proteins are not present in cardiac thick filaments. The structure of the myosin heads in the IHM has a resolution of $12-21 \AA$, which exceeds the current resolution of the IHM in skeletal and cardiac muscle. Taylor and colleagues (Hu et al. 2016) point out that a resolution of $\sim 25 \AA$ or more is needed to determine the structure of the IHM with certainty.

In the Lethocerus IHM, the blocked head interacts with the free head, but not S2, and the free head interacts with the thick filament backbone (Hu et al. 2016; Hu et al. 2017; Taylor et al. 2019). The IHM is perpendicular to the filament axis, and IHMs form the prominent crowns seen at $14.5 \mathrm{~nm}$ spacing in relaxed thick filaments (Fig. 5a). The blocked head projects further from the thick filament than the free head. X-ray diffraction measurements have shown that when fibres are stretched, the helical angle between crowns on the thick filament decreases slightly (Perz-Edwards et al. 2011) and there is indirect evidence that disorder in the blocked head, seen by cryo-electron microscopy, is coupled to the 
change in helical angle in the filament backbone (Hu et al. 2017).

The form of myosin heads in the OFF state of thick filaments, together with X-ray diffraction measurements on fibres during oscillating contractions, has led to a suggestion of how stretch activation might work. Taylor and colleagues (Hu et al. 2017) propose that a rapid stretch might produce disorder in the blocked head, dislodging it from the IHM so that it could bind to a target zone on the thin filament. The blocked head could perform a power stroke, which would pull the free head from the thick filament backbone. Then the free head would bind to a second actin in the same target zone as the blocked head, and perform a power stroke. If there were no other available target zone, the free head would go back to bind to the thick filament backbone, thus limiting the mobility of the blocked head, which would join its partner in an IHM. The disordered blocked head might be a sensor for the state of the thin filament, which would depend on the concentration of $\mathrm{Ca}^{2+}$ in the fibres. Based on

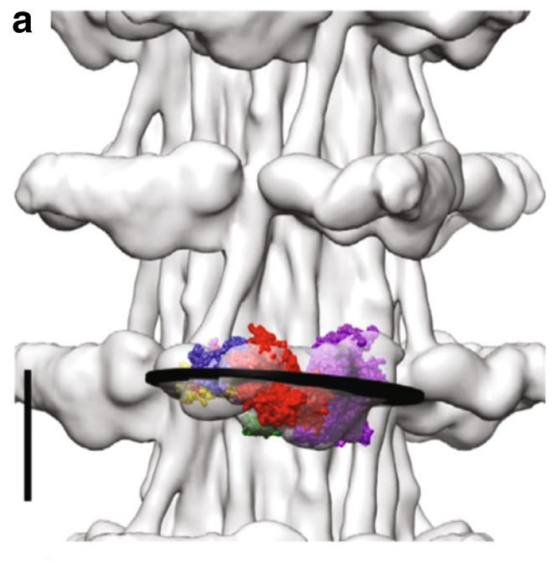

b

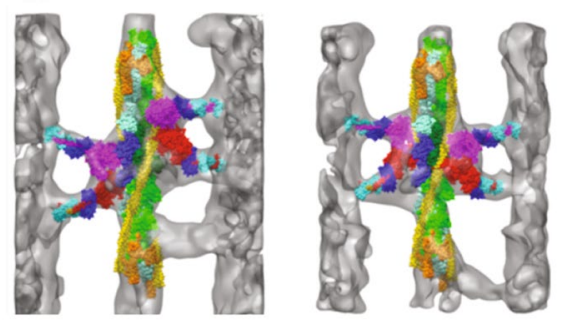

Thick Thin Thick Thick Thin Thick

Fig. 5 The Lethocerus thick filament and troponin bridges. a CryoEM image of a relaxed IFM thick filament. Myosin heads form crowns spaced at $14.5 \mathrm{~nm}$ along the filament. The IHM is nearly perpendicular to the filament axis, with the blocked head projecting furthest from the filament. The bare zone of the filament is at the top. Scale bar $10.0 \mathrm{~nm}$. b Averaged images of IFM during isometric contraction. Crossbridges are fitted with atomic models of a myosin head and $\mathrm{Tn}$ bridges are not. Tn is orange and Tm is yellow. Tn bridges contact the thin filament at or near Tn. a is from Hu et al. (2017); b is from Wu et al. (2010) the ATPase activity of the isolated myosin, the authors calculate that in Lethocerus IFM, the myosin heads could cycle from IHM to active crossbridges at the oscillation frequency of the muscle, which is $30-40 \mathrm{~Hz}$ in vivo. At present this sequence of events is speculative.

\section{Troponin bridges}

Myosin heads bind reversibly to regulated thin filaments. In skeletal and cardiac muscle, binding sites on actin become available when troponin binds $\mathrm{Ca}^{2+}$ and tropomyosin moves from a blocking position on actin. Thin filament activation in these muscles is thought to precede activation of the thick filaments (Irving 2017; Piazzesi et al. 2018). Time-resolved $\mathrm{X}$-ray diffraction measurements of Lethocerus IFM during oscillatory contractions at constant $\mathrm{Ca}^{2+}$ concentration showed changes in the Tm reflection indicating Tm movement in response to stretch, followed by crossbridge binding and development of force; the changes were reversed when the fibres were released (Perz-Edwards et al. 2011). There was evidence for permanent bridges outside the target zone, at the position of $\mathrm{Tn}$; these had been seen previously in electron micrographs of contracting muscle and called Tn bridges (Fig. 5b) (Wu et al. 2010). It was suggested that Tn bridges pull tropomyosin from a blocking position on actin when the muscle is stretched every contraction cycle, and that tropomyosin resumes the blocking position when the muscle is released. The new findings on thick filament regulation in IFM mean that the effect of stretch on the IHM and on Tn bridges must be co-ordinated at contraction frequencies of up to $40 \mathrm{~Hz}$ in Lethocerus and $200 \mathrm{~Hz}$ in Drosophila. This will depend on the compliance of the thick filament backbone as well as that of Tm and Tn bridges.

An inconsistency in thin filament regulation of Lethocerus IFM now has a possible explanation. X-ray diffraction measurements on fibres, in which the length was sinusoidally cycled at a constant priming concentration of $\mathrm{Ca}^{2+}$ (pCa 5.7), showed the intensity of the tropomyosin reflection changing from a maximum at the peak of the stretch to a minimum at the shortest length (where the intensity was equivalent to that of relaxed fibres at pCa 9) (Perz-Edwards et al. 2011). These changes correspond to the transition from the fully open state of the thin filament to the blocked state. Equilibrium and kinetic binding measurements with myosin S1 and thin filaments regulated by Lethocerus Tm-Tn and TnC-F1, as described above, showed that the filaments were about $40 \%$ in the open state (available for myosin binding) and $60 \%$ in the closed state (partially open) at a priming $\mathrm{Ca}^{2+}$ concentration (pCa 6) (Boussouf et al. 2007). Therefore, unstretched fibres would be expected to develop tension under these conditions, but they remain relaxed. However, this might be explained if the thick filaments in 
the fibre diffraction experiments were OFF until stretched. Stress on the Tn bridges (not measurable in the solution studies) would enhance the activation of the thin filaments and promote tropomyosin movement to the blocking position when the stretch was released. The implication is that when thick filaments are activated by stretching at priming $\mathrm{Ca}^{2+}$ concentration, they would find a thin filament already available to bind myosin heads. The function of the unusual TnC-F1 in IFM may be to bias the thin filament towards the partially open state, at the relatively low concentrations of $\mathrm{Ca}^{2+}$ at which TnC-F2 is not active. Tn bridges would act as a hairline trigger to expose actin target sites after a stretch. It is possible that in cardiac fibres, as in IFM, thin filaments are primed for interaction with myosin heads, once the heads are freed from the IHM by stress on the thick filaments at each heartbeat.

\section{Conclusions}

Although IFM has more in common with cardiac muscle than with skeletal muscle, there are many differences between the two. IFM is restricted by the requirement for nearly constant contraction frequency, which is determined by the resonant properties of the thorax, including the muscles. The structure of the IFM is adapted to this requirement: matching periodicities of actin, Tn and target zones on the thin filament, as well as the arrangement of crowns on the thick filament, favour rapid controlled interaction between myosin and actin. Cardiac muscle, which has no such constraints, has a less precise structure.

The more pronounced LDA and SA of IFM, and the small changes in sarcomere length at which the effects are observed, are likely to be a consequence of the high stiffness and regular structure of the IFM sarcomere. The changes in $\mathrm{Ca}^{2+}$ sensitivity and cooperativity produced by MyBP-C have a modulating effect, resulting in some flexibility in the contractile response of cardiac muscle, whereas the same effects produced by TnC-F1 are essential for the function of IFM. At present, it is not clear if MyBP-C and Tn bridges have a comparable function in transmitting force between thick and thin filaments.

The conversion of myosin heads on the thick filament from OFF to ON depends on the force on the filaments. For IFM during oscillatory contractions, the force depends on the load of the wings. This is expected to be constant, so the number of $\mathrm{ON}$ bridges would be the same at each contraction. By contrast, the load on cardiac muscle depends on the arterial pressure and as this varies, the number of $\mathrm{ON}$ bridges will vary. Perhaps the unusual structure of the IHM in Lethocerus IFM is an adaptation to a rapid conversion between states under a constant load.
Thirty years after his experiments with the haltere, Pringle (1978) foresaw the existence of the IHM in thick filaments and Tn bridges in IFM: " $[\ldots]$ the conclusion must be that strain of the myosin filament, produced by stress either through the connecting protein in insect fibrillar muscle or through those cross bridges that are already attached, changes the organization of the myosin filament in some way that makes more bridges available for interaction. What this change of organisation is is completely unknown at the present time, but is likely to be a geometrical effect."

Acknowledgements We are grateful to Dr Robert Edwards and Dr Michael Reedy for Fig. 2b and to Dr Kevin Leonard for Fig. 2d. We would like to thank Dr Alex Stewart for many fruitful discussions. $\mathrm{BB}$ and AP received funds from the BBSRC (BB/M00676X/1). AP received funds from the UK Dementia Research Institute (RE1 3556), which is funded by the MRC, Alzheimer's Society and Alzheimer's Research UK.

Open Access This article is distributed under the terms of the Creative Commons Attribution 4.0 International License (http://creativeco mmons.org/licenses/by/4.0/), which permits unrestricted use, distribution, and reproduction in any medium, provided you give appropriate credit to the original author(s) and the source, provide a link to the Creative Commons license, and indicate if changes were made.

\section{References}

Agianian B, Krzic U, Qiu F, Linke WA, Leonard K, Bullard B (2004) A troponin switch that regulates muscle contraction by stretch instead of $\mathrm{Ca}^{2+}$. EMBO J 23:772-779

Ait-Mou Y, Hsu K, Farman G, Kumar M, Greaser M, Irving T, de Tombe P (2016) Titin strain contributes to the Frank-Starling law of the heart by structural rearrangements of both thin- and thickfilament proteins. Proc Natl Acad Sci USA 113:2306-2311

Alamo L, Wriggers W, Pinto A, Bártoli F, Salazar L, Zhao F, Craig R, Padrón R (2008) Three-dimensional reconstruction of Tarantula myosin filaments suggests how phosphorylation may regulate myosin activity. J Mol Biol 384:780-797

AL-Khayat HA, Hudson L, Reedy MK, Irving TC, Squire JM (2003) Myosin head configuration in relaxed insect flight muscle: X-ray modeled resting cross-bridges in a pre-powerstroke state are poised for actin binding. Biophys J 85(2):1063-1079

Al-Khayat HA, Kensler RW, Squire JM, Marston SB, Morris EP (2013) Atomic model of the human cardiac muscle myosin filament. Proc Natl Acad Sci USA 110:318-323

Blumenschein TMA, Stone DB, Fletterick RJ, Mendelson RA, Sykes $\mathrm{BD}$ (2005) $\mathrm{Ca}^{2+}$-dependent changes in the flexibility of the regulatory domain of Troponin $\mathrm{C}$ in the troponin complex. J Biol Chem 280:21924-21932

Boussouf SE, Agianian B, Bullard B, Geeves MA (2007) The regulation of myosin binding to actin filaments by Lethocerus troponin. J Mol Biol 373:587-598

Bullard B, Pastore A (2011) Regulating the contraction of insect flight muscle. J Muscle Res Cell Motil 32:303-313

Bullard B, Burkart C, Labeit S, Leonard K (2005) The function of elastic proteins in the oscillatory contraction of insect flight muscle. $\mathrm{J}$ Muscle Res Cell Motil 26:479-485

Campbell K, Chandra M (2006) Functions of stretch activation in heart muscle. J Gen Physiol 127:89-94 
Cazorla O, Freiburg A, Helmes M, Centner T, McNabb M, Wu Y, Trombitas K, Labeit S, Granzier H (2000) Differential expression of cardiac titin isoforms and modulation of cellular stiffness. Circ Res 86:59-67

Craig R, Lee KH, Mun JY, Torre I, Luther PK (2014) Structure, sarcomeric organization, and thin filament binding of cardiac myosinbinding protein-C. Pflug Arch 466(3):425-431

De Nicola G, Burkart C, Qiu F, Agianian B, Labeit S, Martin S, Bullard B, Pastore A (2007) The structure of Lethocerus Troponin $\mathrm{C}$ : insights into the mechanism of stretch activation in muscles. Structure 15:813-824

De Nicola GF, Martin S, Bullard B, Pastore A (2010) Solution structure of the Apo C-terminal domain of the Lethocerus F1 Troponin C Isoform. Biochemistry 49:1719-1726

de Tombe PP, Mateja RD, Tachampa K, Ait Mou Y, Farman GP, Irving TC (2010) Myofilament length dependent activation. J Mol Cell Cardiol 48(5):851-858

Dickinson MH, Hyatt J, Lehmann F-O, Moore JR, Reedy MC, Simcox A, Tohtong R, Vigoreaux J, Yamashita H, Maughan DW (1997) Phosphorylation-dependent power output of transgenic flies: an integrated study. Biophys J 73:3122-3134

Dvoretsky A, Abusamhadneh E, Howarth J, Rosevear P (2002) Solution structure of $\mathrm{Ca}^{2+}$-saturated cardiac Troponin $\mathrm{C}$ bound to cardiac Troponin I. J Biol Chem 277:38565-38570

Farman GP, Miller MS, Reedy MC, Soto-Adames FN, Vigoreaux JO, Maughan DW, Irving TC (2009) Phophorylation and the $\mathrm{N}$-terminal extension of the regulatory light chain help orient and align the myosin heads in Drosophila flight muscle. J Struct Biol 128:240-249

Ferguson A, Lakey A, Hutchings A, Butcher GW, Leonard KR, Bullard B (1994) Cytoskeletal proteins of insect muscle: location of zeelins in Lethocerus flight and leg muscle. J Cell Sci 107:1115-1129

Fukuda N, Granzier H, Ishiwata S, Kurihara S (2008) Physiological functions of the giant elastic protein titin in mammalian striated muscle. J Physiol Sci 58:151-159

Fusi L, Brunello E, Yan Z, Irving M (2016) Thick filament mechanosensing is a $\mathrm{Ca}^{2+}$-independent regulatory mechanism in skeletal muscle. Nat Commun 7:13281

Gordon AM, Homsher E, Regnier M (2000) Regulation of contraction in striated muscle. Physiol Rev 80(2):853-924

Harris SP, Belknap B, Van Sciver RE, White HD, Galkin VE (2016) $\mathrm{C} 0$ and $\mathrm{C} 1 \mathrm{~N}$-terminal $\mathrm{Ig}$-domains of myosin binding protein $\mathrm{C}$ exert different effects on thin filament activation. Proc Natl Acad Sci USA 113(6): 1558-1563

Haselgrove J (1975) X-ray evidence for conformational changes in the myosin filaments of vertebrate striated muscle. J Mol Biol 92:113-143

Heeley DH, Belknap B, White HD (2006) Maximal activation of skeletal muscle thin filaments requires both rigor myosin $\mathrm{S} 1$ and calcium. J Biol Chem 281:668-676

Herzberg O, James M (1985) Structure of the $\mathrm{Ca}^{2+}$ regulatory muscle protein Troponin-C at $2.8 \AA$ resolution. Nature 313:653-659

Hooke R (1665) Microscopic observations or wonderful discoveries by the microscope illustrated by thirty-three copper-plates, curiously engraved whereby the most valuable particulars in that celebrated author's are brought together. Robert Wilkinson, London

Houdusse A, Love M, Dominguez R, Grabarek Z, Cohen C (1997) Structures of four $\mathrm{Ca}^{2+}$-bound troponin $\mathrm{C}$ at $2.0 \AA$ resolution: further insights into the $\mathrm{Ca}^{2+}$-switch in the calmodulin superfamily. Structure 5:1695-1711

Houmeida A, Baron A, Keen J, Khan GN, Knight PJ, Stafford WF 3rd, Thirumurugan K, Thompson B, Tskhovrebova L, Trinick J (2008) Evidence for the oligomeric state of 'elastic' titin in muscle sarcomeres. J Mol Biol 384(2):299-312
Hu Z, Taylor DW, Reedy MK, Edwards RJ, Taylor KA (2016) Structure of myosin filaments from relaxed Lethocerus flight muscle by cryo-EM at $6 \AA$ resolution. Sci. Adv. 2:e1600058

Hu Z, Taylor DW, Edwards RJ, Taylor KA (2017) Coupling between myosin head conformation and the thick filament backbone structure. J Struct Biol 200:334-342

Irving M (2017) Regulation of contraction by the thick filaments in skeletal muscle. Biophys J 113:2579-2594

Josephson RK, Malamud JG, Stokes DR (2000) Asynchronous muscle: a primer. J Exp Biol 203(Pt 18):2713-2722

Kampourakis T, Yan Z, Gautel M, Sun Y-B, Irving M (2014) Myosin binding protein-C activates thin filaments and inhibits thick filaments in heart muscle cells. Proc Natl Acad Sci USA 111:18763-18768

Kampourakis T, Sun Y-B, Irving M (2016) Myosin light chain phosphorylation enhances contraction of heart muscle via structural changes in both thick and thin filaments. Proc Natl Acad Sci USA 113:E3039-E3047

Kolmerer B, Clayton J, Benes V, Allen T, Ferguson C, Leonard K, Weber U, Knekt M, Ansorge W, Labeit S, Bullard B (2000) Sequence and expression of the kettin gene in Drosophila melanogaster and Caenorhabditis elegans. J Mol Biol 296:435-448

Krüger M, Linke W (2011) The giant protein titin: a regulatory node that integrates myocyte signaling pathways. J Biol Chem 286:9905-9912

Krzic U, Rybin V, Leonard KR, Linke WA, Bullard B (2010) Regulation of oscillatory contraction in insect flight muscle by troponin. J Mol Biol 397:110-118

Kulke M, Neagoe C, Kolmerer B, Minajeva A, Hinssen H, Bullard B, Linke WA (2001) Kettin, a major source of myofibrillar stiffness in Drosophila indirect flight muscle. J Cell Biol 154(5):1045-1057

Labeit S, Gautel M, Lakey A, Trinick J (1992) Towards a molecular understanding of titin. EMBO J 11:1711-1716

Leake M, Wilson D, Bullard B, Simmons R (2003) The elasticity of single kettin molecules using a two-bead laser-tweezers assay. FEBS Lett 535:55-60

Levine RJ, Kensler RW, Yang Z, Stull JT, Sweeney HL (1996) Myosin light chain phosphorylation affects the structure of rabbit skeletal muscle thick filaments. Biophys J 71:898-907

Linari M, Reedy MK, Reedy MC, Lombardi V, Piazzesi G (2004) Caactivation and stretch-activation in insect flight muscle. Biophys J 87:1101-1111

Linari M, Brunello E, Reconditi M, Fusi L, Caremani M, Narayanan T, Piazzesi G, Lombardi V, Irving M (2015) Force generation by skeletal muscle is controlled by mechanosensing in myosin filaments. Nature 528:276-279

Lindhout D, Sykes B (2003) Structure and dynamics of the C-domain of human cardiac Troponin $\mathrm{C}$ in complex with the inhibitory region of human cardiac Troponin I. J Biol Chem 278:27024-27034

Linke WA (2007) Sense and stretchability: the role of titin and titinassociated proteins in myocardial stress-sensing and mechanical dysfunction. Cardiovasc Res 77(4):637-648

Linke WA (2018) Titin gene and protein functions in passive and active muscle. Ann Rev Physiol 80:389-411

Lowey S, Saraswat LD, Liu H, Volkman N, Hanein D (2007) Evidence for an interaction between the SH3 domain and the N-terminal extension of the essential light chain in class II myosins. J Mol Biol 371:902-913

Martin S, Avella G, Adrover M, de Nicola G, Bullard B, Pastore A (2011) Binding properties of the $\mathrm{Ca}^{2+}$-activated $\mathrm{F} 2$ isoform of Lethocerus troponin C. Biochemistry 50:1839-1847

McKillop DF, Geeves MA (1993) Regulation of the interaction between actin and myosin subfragment 1: evidence for three states of the thin filament. Biophys J 65:693-701 
McNamara JW, Li A, Smith NJ, Lal S, Graham RM, Bezold Kooiker K, van Dijk SJ, dos Remedios CG, Harris SP, Cooke R (2016) Ablation of cardiac myosin binding protein-C disrupts the superrelaxed state of myosin in murine cardiomyocytes. J Mol Cardiol 94:65-71

Miller A, Tregear R (1972) Structure of insect fibrillar flight muscle in the presence and absence of ATP. J Mol Biol 70:85-104

Miller M, Granzier H, Ehler E, Gragorio CC (2004) The sensitive giant: the role of titin-based stretch sensing complexes in the heart. Trends Cell Biol 14:119-126

Moore JR, Dickinson MH, Vigoreaux JO, Maughan DW (2000) The effect of removing the N-terminal extension of the Drosophila myosin regulatory light chain upon flight ability and contractile dynamics of indirect flight muscle. Biophys J 78:1431-1440

Morris EP, Squire JM, Fuller GW (1991) The 4-stranded helical arrangement of myosin heads on insect (Lethocerus) flight muscle thick filaments. J Struct Biol 107:237-249

Mun JY, Previs MJ, Craig R (2014) Myosin-binding protein C displaces tropomyosin to activate cardiac thin filaments and governs their speed by an independent mechanism. Proc Natl Acad Sci USA 111:2170-2175

Neagoe C, Opitz CA, Makarenko I, Linke WA (2003) Gigantic variety: expression patterns of titin isoforms in striated muscles and consequences for myofibrillar passive stiffness. J Muscle Res Cell Motil 24:175-179

Perz-Edwards RJ, Irving TC, Baumann BA, Gore D, Hutchinson DC, Krzic U, Porter RL, Ward AB, Reedy MK (2011) X-ray diffraction evidence for myosin-troponin connections and tropomyosin movement during stretch activation of insect flight muscle. Proc Natl Acad Sci USA 108(1):120-125

Pfuhl M, Gautel M (2012) Structure, interactions and function of the $\mathrm{N}$-terminus of cardiac myosin binding protein $\mathrm{C}$ (MyBP-C): who does what, with what, and to whom? J Muscle Res Cell Motil 33:83-94

Piazzesi G, Caremani M, Linari M, Reconditi M, Lombardi V (2018) Thick filament mechano-sensing in skeletal and cardiac muscles: a common mechanism able to adapt the energetic cost of the contraction to the task. Front Physiol 9:1-7

Pringle J (1949) The excitation and contraction of the flight muscles of insects. J Physiol 108:226-232

Pringle JW (1978) The Croonian Lecture (1977). Stretch activation of muscle: function and mechanism. Proc R Soc Lond B 201:107-130

Qiu F, Brendel S, Cunha PMF, Astola N, Song B, Furlong EEM, Leonard KR, Bullard B (2005) Myofilin, a protein on the thick filaments of insect muscle. J Cell Sci 118:1527-1536

Reconditi M, Brunello E, Fusi L, Linari M, Martinez MF, Lombardi V (2014) Sarcomere-length dependence of myosin filament structure in skeletal muscle fibres of the frog. J Physiol 592:1119-1137

Reconditi M, Caremani M, Pinzauti F, Powers JD, Narayanan T, Stienen GJ et al (2017) Myosin filament activation in the heart is tuned to the mechanical task. Proc Natl Acad Sci USA 114:3240-3245

Reedy MK, Reedy MC (1985) Rigor crossbridge structure in tilted single filament layers and flared-X formations from insect flight muscle. J Mol Biol 185:145-176

Reedy MK, Leonard KR, Freeman R, Arad T (1981) Thick filament mass determination by electron scattering measurements with the scanning transmission electron microscope. J Muscle Res Cell Motil 2:45-64

Reedy MC, Reedy MK, Leonard KR, Bullard B (1994) Gold/Fab immuno electron microscopy localization of Troponin $\mathrm{H}$ and Troponin T in Lethocerus flight muscle. J Mol Biol 239(1):52-67

Risi C, Eisner J, Belknap B, Heeley DH, White HD, Schröder GF, Galkin VE (2017) $\mathrm{Ca}^{2+}$-induced movement of tropomyosin on native cardiac thin filaments revealed by cryoelectron microscopy. Proc Natl Acad Sci USA 114(26):6782-6787

Ruiz T, Bullard B, Lepault J (1998) Effects of $\mathrm{Ca}^{2+}$ and nucleotides on the structure of insect flight muscle thin filaments. J Muscle Res Cell Motil 19(4):353-364

Sanfelice D, Sanz-Hernández M, de Simone A, Bullard B, Pastore A (2016) Toward understanding the molecular bases of stretch activation. J Biol Chem 291:16090-16099

Satyshur KA, Rao ST, Pyzalska D, Drendel W, Greaser M, Sundaralingam M (1988) Refined structure of chicken skeletal muscle troponin $\mathrm{C}$ in the two- $\mathrm{Ca}^{2+}$ state at 2-A resolution. J Biol Chem 263:1628-1647

Schmitz H, Lucaveche C, Reedy MK, Taylor KA (1994) Oblique section 3-D reconstruction of relaxed insect flight muscle reveals cross bridge lattice in helical registration. Biophys $\mathrm{J}$ 67(4):1620-1633

Sia S, Li M, Spyracopoulos L, Gagné S, Liu W, Putkey J, Sykes B (1997) Structure of cardiac muscle Troponin C unexpectedly reveals a closed regulatory domain. J Biol Chem 272:18216-18221

Slupsky C, Sykes B (1995) NMR solution structure of $\mathrm{Ca}^{2+}$-saturated skeletal muscle Troponin C. Biochemistry 34:15953-15964

Stelzer JE, Moss RL (2006) Contributions of stretch activation to length-dependent contraction in murine myocardium. J Gen Physiol 128:461-471

Stelzer J, Larsson L, Fitzsimons D, Moss R (2006a) Activation dependence of stretch activation in mouse skinned myocardium: implications for ventricular function. J Gen Physiol 127:95-107

Stelzer JE, Patel JR, Moss RL (2006b) Acceleration of stretch activation in murine myocardium due to phosphorylation of myosin regulatory light chain. J Gen Physiol 128:261-272

Stewart MA, Franks-Skiba K, Chen S, Cooke R (2010) Myosin ATP turnover rate is a mechanism involved in thermogenesis in resting skeletal muscle fibers. Proc Natl Acad Sci USA 107:430-435

Stull JT, Kamm KE, Vandenboom R (2011) Myosin light chain kinase and the role of myosin light chain phosphorylation in skeletal muscle. Arch Biochem Biophys 510:120-128

Sulbarán G, Biasutto A, Alamo L, Riggs C, Pinto A, Méndez F, Craig R, Padrón R (2013) Different head environments in Tarantula thick filaments support a cooperative activation process. Biophys J 105:2114-2122

Taylor KA, Rahmani H, Edwards RJ, Reedy MK (2019) Insights into actin-myosin interactions within muscle from 3D electron microscopy. Int J Mol Sci 20:1703-1734

Tohtong R, Yamashita H, Graham M, Haeberle J, Simcox A, Maughan D (1995) Impairment of muscle function caused by mutations of phosphorylation sites in myosin regulatory light chain. Nature 374:650-653

Tonino P, Kiss B, Strom J, Methawasin M, Smith JE 3rd, Kolb J, Labeit S, Granzier H (2017) The giant protein titin regulates the length of the striated muscle thick filament. Nat Commun 8(1):1041-1052

Trayer IP, Trayer HR, Levine BA (1987) Evidence that the N-terminal region of A1-light chain of myosin interacts directly with the C-terminal region of actin. A proton magnetic resonance study. Eur J Biochem 164:259-266

Tregear RT, Reedy MC, Goldman YE, Taylor KA, Winkler H, FranziniArmstrong C, Sasaki H, Lucaveche C, Reedy M (2004) Crossbridge number, position, and angle in target zones of cryofixed isometrically active insect flight muscle. Biophys J 86:3009-3019

Trinick J (1996) Titin as a scaffold and a spring. Curr Biol 6:258-260

Tsuda S, Miura A, Gagné S, Spyracopoulos L, Sykes B (1999) Low-temperature-induced structural changes in the apo regulatory domain of skeletal muscle Troponin C. Biochemistry 38:5693-5700

van Leeuwenhoek A (1719) Opera Omina. Johannes Arnold Langerak, Leiden 
Vinogradova MV, Stone DB, Malanina GG, Kartzaferi C, Cooke R, Mendelson RA, Fletterick RJ (2005) $\mathrm{Ca}^{2+}$-regulated structural changes in troponin. Proc Natl Acad Sci USA 102:5038-5043

Woodhead JL, Zhao FQ, Craig R, Egelman EH, Alamo L, Padrón R (2005) Atomic model of a myosin filament in the relaxed state. Nature 436:1195-1199

Wu S, Liu J, Reedy MC, Tregear RT, Winkler H, Franzini-Armstrong C, Sasaki H, Lucaveche C, Goldman YE, Reedy MK, Taylor KA (2010) Electron tomography of cryofixed, isometrically contracting insect flight muscle reveals novel actin-myosin interactions. PLoS ONE 5(9):e12643
Zoghbi ME, Woodhead JL, Moss RL, Craig R (2008) Three-dimensional structure of vertebrate cardiac muscle myosin filaments. Proc Natl Acad Sci USA 105:2386-2390

Publisher's Note Springer Nature remains neutral with regard to jurisdictional claims in published maps and institutional affiliations. 